\title{
Neutrino Helicity Reversal and Fundamental Symmetries
}

\author{
U. D. Jentschura ${ }^{1,2}$ and B. J. Wundt ${ }^{1}$ \\ ${ }^{1}$ Department of Physics, Missouri University of Science and Technology, Rolla, Missouri 65409, USA \\ ${ }^{2}$ MTA-DE Particle Physics Research Group, P.O.Box 51, H-4001 Debrecen, Hungary
}

\begin{abstract}
A rather elusive helicity reversal occurs in a gedanken experiment in which a massive left-handed Dirac neutrino, traveling at a velocity $u<c$, is overtaken on a highway by a speeding vehicle (traveling at velocity $v$ with $u<v<c$ ). Namely, after passing the neutrino, looking back, one would see a right-handed neutrino (which has never been observed in nature). The Lorentz-invariant mass of the right-handed neutrino is still the same as before the passing. The gedanken experiment thus implies the existence of right-handed, light neutrinos, which are not completely sterile. Furthermore, overtaking a bunch of massive right-handed Dirac neutrinos leads to gradual de-sterilization. We discuss the helicity reversal and the concomitant sterilization and de-sterilization mechanisms by way of an illustrative example calculation, with a special emphasis on massive Dirac and Majorana neutrinos. We contrast the formalism with a modified Dirac neutrino described by a Dirac equation with a pseudoscalar mass term proportional to the fifth current.
\end{abstract}

PACS numbers: 95.85.Ry, 11.10.-z, 03.70.+k

\section{INTRODUCTION}

The neutrino (here collectively used in order to denote a mass eigenstate of the neutrino field) is the only particle in the (extended) Standard Model for which an appropriate first-quantized description has not yet been completely determined, and the observation of nonvanishing neutrino masses has not simplified the task (for pertinent overview, see Refs. [1 4]). Possible candidate equations include (i) the Dirac equation with a standard, scalar mass term [5], (ii) the Majorana equation (which would imply that neutrinos are equal to their own antiparticle, see Ref. [6]), and (iii) generalized Dirac equations with a pseudoscalar mass term [7-13]. The latter equation leads to a manifest helicity dependence of the anti-commutators of the field operators and to a superluminal (tachyonic) dispersion relation, which has recently been discussed in Ref. [13]. Recent claims regarding a possibly superluminal nature of the neutrino, which eventually turned out to be in error, have inspired theorists to revisit theoretical ideas whose relevance extends beyond relevance to the experimental claims. However, in order to keep things in perspective, we should remember that the origin of these ideas dates back about three decades [7]. Finally, (iv) further modified Dirac equations with small Lorentz-breaking terms have also been discussed in the literature [14].

In the current article, our focus will be on a comparison of the helicity suppression in models (i) and (iii), while the Majorana character of a neutrino (ii) is commonly associated to the seesaw mechanism [15 18], which generates neutrino masses and the mass gap of left-handed and right-handed neutrinos by spontaneous symmetry breaking. It leads to an effective suppression of the right-handed neutrino interaction, in view of a large mass separation of the different helicities. Namely, right-handed neutrinos acquire a mass of order $\Lambda_{\mathrm{GUT}}$, where $\Lambda_{\mathrm{GUT}}$ is the grand unification scale, while left-handed neutrinos acquire a mass of the order of $v^{2} / \Lambda_{\mathrm{GUT}}$, where $v$ is the Higgs vacuum expectations value. From the point of view of fundamental symmetries, a Majorana character of the neutrino, combined with the seesaw mechanism, would solve two issues simultaneously. Namely, (i) it would explain why the neutrino masses are so small as compared to the masses of other particles in the Standard Model, and (ii) it would offer a very natural interpretation for the elusive helicity reversal of a neutrino overtaken by a speeding vehicle on a highway. The righthanded state would naturally be interpreted as a right-handed anti-neutrino, i.e., as a physical state of the neutrino's own (identical) anti-particle.

However, the introduction of a Majorana neutrino into an extended Standard Model is not as innocent as it seems. In particular, the Majorana mass term violates lepton number, which is a global symmetry that tracks the difference between particles and antiparticles (an excellent overview is presented in Ref. [6]). While there is nothing sacred about global symmetries, "lepton number conservationalists" might find the lack of a proper distinction of particles and antiparticles disturbing. A Majorana neutrino would be the only spin- $\frac{1}{2}$ particle in an (extended) Standard Model which is equal to its own antiparticle (the original standard model called for a Weyl neutrino). Furthermore, a Majorana neutrino is described by an equation which, on the level of first quantization, does not allow plane-wave solutions of the form $u \exp (-\mathrm{i} k \cdot x)$, where $u$ is a spinor (or polarization vector in the case of spin- 1 particles) and $k \cdot x=E t-\vec{k} \cdot \vec{r}$ is the scalar product of energy-momentum and space-time coordinate (see also C). Recent measurements set rather strict bounds for the magnitude of the Majorana mass terms [19 22] as inferred from neutrinoless double beta decay experiments.

In order to put things into perspective, we recall that in the "good old times", neutrinos were supposed to be massless 
Weyl fermions which come in only one helicity state. Weyl fermions transform according to the fundamental $\left(\frac{1}{2}, 0\right)$ representation of the Lorentz group. The well-known discovery of neutrino oscillations implies that the neutrinos need to have a nonvanishing mass. If neutrinos are Majorana fermions, then the right-handed states could be interpreted as antineutrinos because Majorana fermions are equal to their own antiparticles, but this interpretation is unavailable if neutrinos are Dirac fermions. Because of the historical, proverbial association of very high speeds with the "autobahn", we would like to refer to the underlying question as the "helicity reversal paradox" or even the "autobahn helicity paradox" while stressing that the problem has been raised here before in the scientific literature. Other possible designations, inspired by Ref. [23], would include the "rabbit paradox" in light of the fact that high propagating velocities needed to overtake a neutrino are commonly associated with the physical abilities of a species known as the "lepus" or "lepus curpaeums" (in Latin). Indeed, the elusive helicity reversal of a neutrino, overtaken on a highway by speeding vehicle, has given rise to a few questions discussed by Goldhaber and Goldhaber [24]. In Ref. 25] (labelled as "question \#76" regarding "neutrino mass and helicity" in the American Journal of Physics), the author raises the question as to how the helicity flip upon (hypothetically) overtaking a left-handed neutrino should be interpreted physically: If only left-helicity neutrino states take part in the weak interaction and helicity is not conserved upon a Lorentz boost, then is the theory of the weak interaction self-consistent? Although this paradox has been around for as long as theorists have conceived of massive neutrinos and has often been discussed and argued in qualitative terms, it is somewhat surprising that we were unable to find a direct quantitative treatment of the relevant physics in the academic literature. We note that helicity (not chirality!) remains a good quantum number for a massive neutrino. An attempt to address the questions was presented in Ref. [26]. In the helicity basis (see Chap. 23 of Ref. [27], Sec. 2.8.1 on p. 28 of Ref. 2] and Sec. 3 of Ref. [13]), plane-wave neutrino states are characterized by the wave vector $\vec{k}$ and the helicity quantum number $\sigma$. The wave vector $\vec{k}$ constitutes a quantum number in a continuous spectrum. In general, the energy $E \rightarrow E^{\prime}$, the wave vector $\vec{k} \rightarrow \vec{k}^{\prime}$ and also the helicity $\sigma \rightarrow \sigma^{\prime}$ change upon a Lorentz boost (transformation to a different Lorentz frame). In particular, the helicity quantum number $\sigma$ reverses sign ("flips") as one "overtakes" the neutrino, by going into a reference frame which travels at a velocity $v>u$ in the direction of the neutrino velocity $u<c$ (one has $u<v<c$ ). A right-handed (helicity) Dirac neutrino is not completely sterile because the projector $\gamma^{\mu}\left(1-\gamma^{5}\right)$ in the weak-interaction Lagrangian projects onto left-handed chirality, not helicity, states, and right-handed (helicity) massive subluminal Dirac neutrinos are never in a perfect right-handed chirality eigenstate.

Here, we start with a discussion of the helicity suppression for a standard Dirac neutrino in Sec. [II] The case of a Majorana neutrino is discussed in Sec. III. The necessary formalism for tachyonic Dirac neutrinos is recalled in Sec. IV] We show that real as opposed to virtual spin- $1 / 2$ particles described by the tachyonic Dirac equation are always left-handed. Conclusions are given in Sec. $\mathrm{V}$ while $\mathrm{A}$ is devoted to a discussion of the transformation into a rotating frame of reference. Further physical consequences of a neutrino theory based on the generalized Dirac equation are discussed in $\mathrm{B}$. Finally, $\mathrm{C}$ is devoted to the Majorana equation. Units with $\hbar=c=\epsilon_{0}=1$ are used throughout this article.

\section{DIRAC EQUATION AND HELICITY SUPPRESSION}

We start from the standard Dirac equation with a scalar mass term,

$$
\left(\mathrm{i} \gamma^{\mu} \partial_{\mu}-m\right) \psi(x)=0, \quad x^{\mu}=(t, \vec{r}),
$$

where the matrices are used in the standard (Dirac) representation,

$$
\gamma^{0}=\beta=\left(\begin{array}{cc}
\mathbf{1}_{2 \times 2} & 0 \\
0 & -\mathbf{1}_{2 \times 2}
\end{array}\right), \quad \gamma^{i}=\left(\begin{array}{cc}
0 & \sigma^{i} \\
-\sigma^{i} & 0
\end{array}\right), \quad \gamma^{5}=\left(\begin{array}{cc}
0 & \mathbf{1}_{2 \times 2} \\
\mathbf{1}_{2 \times 2} & 0
\end{array}\right) .
$$

We define $\alpha^{i}=\gamma^{0} \gamma^{i}$ and $\beta=\gamma^{0}$, with Latin as opposed to Greek superscripts indicating the spatial components. The Lagrangian density is $\mathcal{L}=\bar{\psi}(x)\left(\mathrm{i} \gamma^{\mu} \partial_{\mu}-m\right) \psi(x)$, and the Hamiltonian is Hermitian, $H=\vec{\alpha} \cdot \vec{p}+\beta m$. The fundamental positive-energy plain-wave eigenspinors are given by $\psi(x)=u_{ \pm}(\vec{k}) \mathrm{e}^{-\mathrm{i} k \cdot x}$ with $k \cdot x=E t-\vec{k} \cdot \vec{r}$ and

$$
u_{+}(\vec{k})=\left(\begin{array}{c}
\sqrt{\frac{E+m}{2 m}} a_{+}(\vec{k}) \\
\sqrt{\frac{E-m}{2 m}} a_{+}(\vec{k})
\end{array}\right), \quad u_{-}(\vec{k})=\left(\begin{array}{c}
\sqrt{\frac{E+m}{2 m}} a_{-}(\vec{k}) \\
-\sqrt{\frac{E-m}{2 m}} a_{-}(\vec{k})
\end{array}\right) .
$$

We here use the covariant normalization $\bar{u}_{\sigma}(\vec{k}) u_{\sigma}(\vec{k})=u_{\sigma}^{+}(\vec{k}) \gamma^{0} u_{\sigma}(\vec{k})=1$, which is different from Chap. 23 of Ref. [27] and Sec. 2.8.1 on p. 28 of Ref. [2]. The energy is $E=\sqrt{\vec{k}^{2}+m^{2}}$. Negative-energy eigenstates of the Dirac 


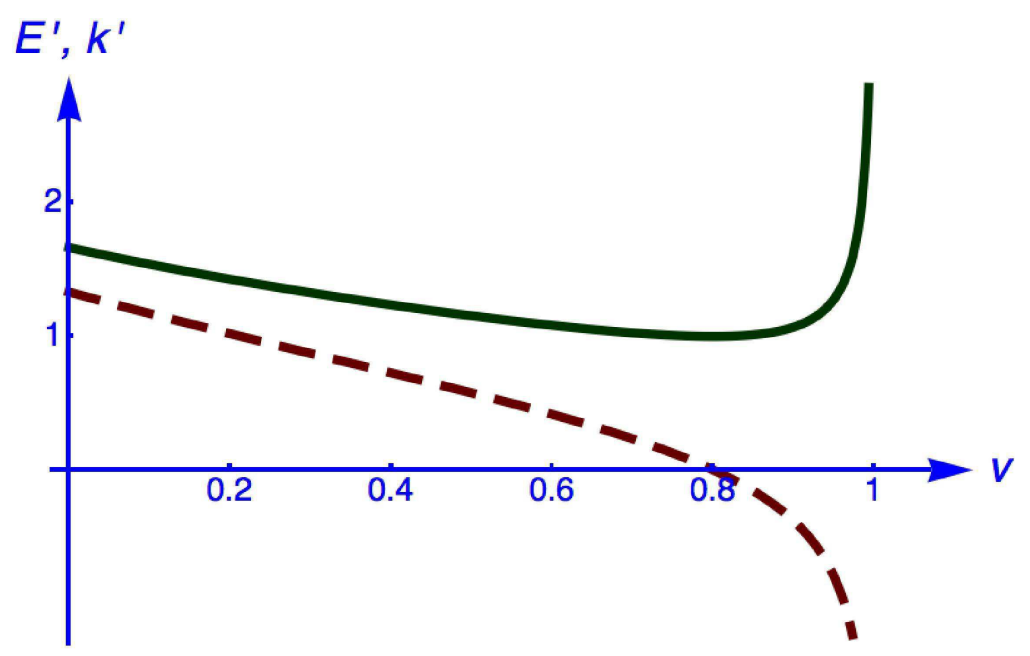

FIG. 1: Illustration of Eq. (14). The Lorentz-transformed energy $E^{\prime}$ is plotted (solid curve) for $u=0.8$, as seen in a frame of reference moving parallel to the $z$ axis at velocity $v$ (measured as a fraction of the speed of light). The energy attains a normalized value $E^{\prime}=1$ when the particle is at rest in the moving frame (i.e., for $v=u$ ). For $v>u$, the particle is overtaken and gains energy in the moving frame, while the $z$ component of the Lorentz-transformed momentum $k^{\prime}$ changes sign (dashed curve).

equation in the helicity basis are given as $\psi(x)=v_{ \pm}(\vec{k}) \mathrm{e}^{\mathrm{i} k \cdot x}$ with

$$
v_{+}(\vec{k})=\left(\begin{array}{l}
-\sqrt{\frac{E-m}{2 m}} a_{+}(\vec{k}) \\
-\sqrt{\frac{E+m}{2 m}} a_{+}(\vec{k})
\end{array}\right), \quad v_{-}(\vec{k})=\left(\begin{array}{c}
-\sqrt{\frac{E-m}{2 m}} a_{-}(\vec{k}) \\
\sqrt{\frac{E+m}{2 m}} a_{-}(\vec{k})
\end{array}\right) .
$$

The normalization is $\bar{v}_{\sigma}(\vec{k}) v_{\sigma}(\vec{k})=-1$. The $a_{\sigma}(\vec{k})$ are the fundamental helicity spinors, where the quantum number $\sigma= \pm$ is equal to the helicity for positive-energy states, and equal to the opposite helicity for negative-energy states. In spherical coordinates, we have

$$
a_{+}(\vec{k})=\left(\begin{array}{c}
\cos \left(\frac{\theta}{2}\right) \\
\sin \left(\frac{\theta}{2}\right) \mathrm{e}^{\mathrm{i} \varphi}
\end{array}\right), \quad a_{-}(\vec{k})=\left(\begin{array}{c}
-\sin \left(\frac{\theta}{2}\right) \mathrm{e}^{-\mathrm{i} \varphi} \\
\cos \left(\frac{\theta}{2}\right)
\end{array}\right)
$$

Here, $\theta$ and $\varphi$ are the polar and azimuthal angles of the wave vector $\vec{k}$; they of course do not depend on the coordinate vector $\vec{r}$. The eigenspinors fulfill the following projector sum rules,

$$
\sum_{\sigma} u_{\sigma}(\vec{k}) \otimes \bar{u}_{\sigma}(\vec{k})=\frac{\not k+m}{2 m}, \quad \sum_{\sigma} v_{\sigma}(\vec{k}) \otimes \bar{v}_{\sigma}(\vec{k})=\frac{\not k-m}{2 m}
$$

We now consider in detail the transformation of the bispinor wave function under a Lorentz boost, which we denote by a change in the reference frame from the unprimed to the primed coordinate system. Namely, under a Lorentz transformation $\Lambda$ with spinor representation $S(\Lambda)$, the bispinor wave function transforms as $\psi^{\prime}\left(x^{\prime}\right)=S(\Lambda) \psi(x)$, with

$$
\begin{aligned}
\psi(x) & =u_{\sigma}(\vec{k}) \exp (-\mathrm{i} k \cdot x) \rightarrow \psi^{\prime}\left(x^{\prime}\right)=S(\Lambda) \psi(x)=u_{\sigma^{\prime}}\left(\vec{k}^{\prime}\right) \exp \left(-\mathrm{i} k^{\prime} \cdot x^{\prime}\right), \\
u_{\sigma^{\prime}}\left(\vec{k}^{\prime}\right) & =S(\Lambda) u_{\sigma}(\vec{k})
\end{aligned}
$$

The Dirac representation is recovered in the moving frame,

$$
\gamma^{\prime \mu}=\Lambda_{\nu}^{\mu} S(\Lambda) \gamma^{\nu} S(\Lambda)^{-1}=\gamma^{\mu}, \quad \Lambda_{\nu}^{\mu} \gamma^{\nu}=S(\Lambda)^{-1} \gamma^{\mu} S(\Lambda)
$$

where the latter relation is obtained from the former by the interchange $\mu \leftrightarrow \nu$ and multiplication by $S(\Lambda)^{-1}$ from the right and by $S(\Lambda)$ from the left. We briefly verify that this is consistent with the transformation of the theorem 


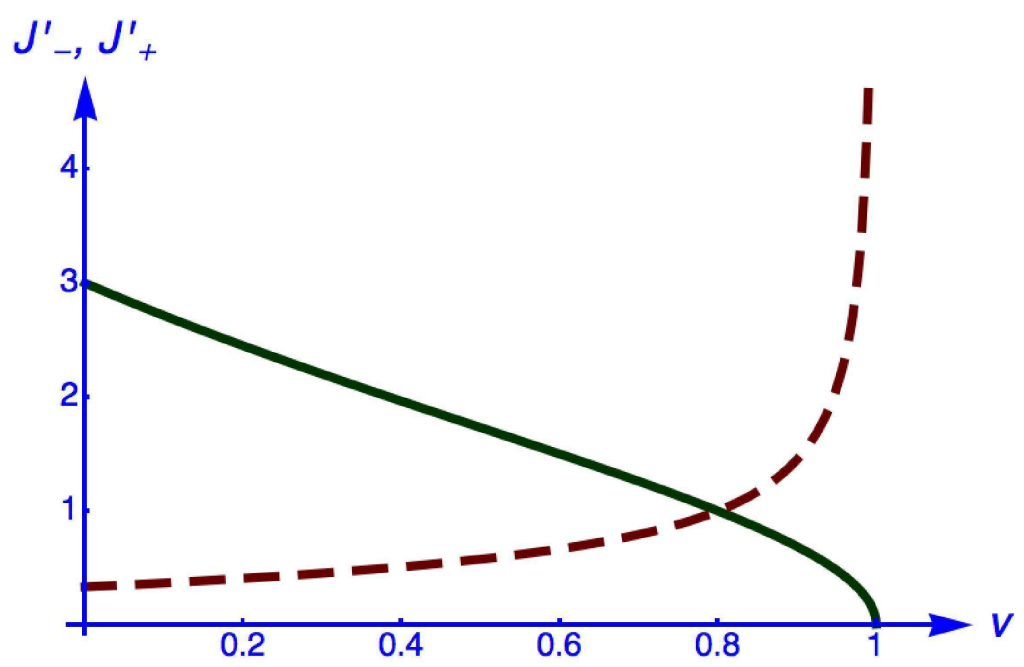

FIG. 2: Illustration of Eq. (17). The Lorentz-transformed current amplitude $J_{-}^{\prime}$, which corresponds to an (initially) left-handed neutrino, is gradually sterilized as the neutrino is overtaken (solid curve), and the helicity flips (we use a value of $u=0.8$ ). Conversely, the current amplitude $J_{+}^{\prime}$, for an initially right-handed neutrino (dashed curve) is gradually de-sterilized as the neutrino is overtaken. The two curves cross at $v=u$, which is the moment where the neutrino is being passed and the helicity flips. Neither the left-handed nor the right-handed standard Dirac neutrino are ever completely sterile, although $J_{-}^{\prime} \rightarrow 0$ as $v \rightarrow 1$. Furthermore, because the mass of the neutrino is Lorentz invariant, both helicity states correspond to very light fermions with the same mass as the left-handed neutrinos.

$\bar{u}_{\sigma}(k) \gamma^{\mu} u_{\sigma}(k)=k^{\mu} / m$ under a Lorentz boost,

$$
\begin{aligned}
\bar{u}_{\sigma^{\prime}}\left(k^{\prime}\right) \gamma^{\mu} u_{\sigma^{\prime}}\left(k^{\prime}\right) & =\bar{u}_{\sigma}(k) S(\Lambda)^{-1} \gamma^{\mu} S(\Lambda) u_{\sigma}(k) \\
& =\Lambda_{\nu}^{\mu}{ }_{\nu} \bar{u}_{\sigma}(k) \gamma^{\nu} u_{\sigma}(k)=\Lambda^{\mu}{ }_{\nu} \frac{k^{\nu}}{m}=\frac{k^{\prime \mu}}{m} .
\end{aligned}
$$

In regard to the elusive helicity flip $\underline{24}[26]$, it is worthwhile to consider the current amplitude

$$
J_{\sigma}^{\mu}=\bar{u}_{\sigma}(\vec{k}) \gamma^{\mu}\left(1-\gamma^{5}\right) u_{\sigma}(\vec{k}),
$$

which describes the forward scattering of a positive-energy neutrino in the helicity state $\sigma$ and with wave vector $\vec{k}$. Under a Lorentz transformation $\Lambda^{\mu}{ }_{\nu}$ with corresponding spinor transformation $S(\Lambda)$, this amplitude transforms into

$$
J_{\sigma}^{\prime \mu}=\Lambda_{\nu}^{\mu}{ }_{\nu} \bar{u}_{\sigma}(\vec{k}) \gamma^{\nu}\left(1-\gamma^{5}\right) u_{\sigma}(k)=\bar{u}_{\sigma^{\prime}}\left(\vec{k}^{\prime}\right) \gamma^{\mu}\left(1-\gamma^{5}\right) u_{\sigma^{\prime}}\left(\vec{k}^{\prime}\right)
$$

For a Lorentz boost in the $z$ direction, we have

$$
\begin{aligned}
\Lambda^{\mu}{ }_{\nu} & =\left(\begin{array}{cccc}
\cosh (\rho) & 0 & 0 & -\sinh (\rho) \\
0 & 1 & 0 & 0 \\
0 & 0 & 1 & 0 \\
-\sinh (\rho) & 0 & 0 & \cosh (\rho)
\end{array}\right), \\
S(\Lambda) & =\left(\begin{array}{cccc}
\cosh \left(\frac{1}{2} \rho\right) & 0 & -\sinh \left(\frac{1}{2} \rho\right) & 0 \\
0 & \cosh \left(\frac{1}{2} \rho\right) & 0 & \sinh \left(\frac{1}{2} \rho\right) \\
-\sinh \left(\frac{1}{2} \rho\right) & 0 & \cosh \left(\frac{1}{2} \rho\right) & 0 \\
0 & \sinh \left(\frac{1}{2} \rho\right) & 0 & \cosh \left(\frac{1}{2} \rho\right)
\end{array}\right),
\end{aligned}
$$

where $\rho$ is the rapidity and $\gamma=1 / \sqrt{1-v^{2}}=\cosh (\rho)$, whereas $\gamma v=\sinh (\rho)$. We note that $S(\Lambda)$ acts in the space of bispinors, whereas $\Lambda^{\mu}{ }_{\nu}$ acts in the space of Lorentz vectors, and that the matrix representation of $S(\Lambda)$ is tied to the Dirac representation which we use for the $\gamma$ matrices. Surprisingly, explicit expressions for spinor Lorentz boosts in the Dirac representation of the Dirac algebra seem to be rather scarce in the literature; our formula is in agreement with the discussion in Ref. [28] and with Eq. (2.74) in Sec. 2.4.1 on p. 15 of Ref. [2]. Note that $S(\Lambda)$ would read 
differently in the chiral representation of the Dirac matrices. Irrespective of the matrix representation of the Dirac algebra, we have

$$
S(\Lambda)=\exp \left(-\frac{\mathrm{i}}{4} \sigma^{\alpha \beta} \omega_{\alpha \beta}\right), \quad \sigma^{\alpha \beta}=\frac{\mathrm{i}}{2}\left[\gamma^{\alpha}, \gamma^{\beta}\right], \quad \omega_{03}=-\omega_{30}=-\rho
$$

while all other elements $\omega_{\mu \nu}$ vanish. We assume that $\vec{k}=k \hat{e}_{z}$ points into the positive $z$ direction, while the Lorentzboosted $\vec{k}^{\prime}=k^{\prime} \hat{e}_{z}$ may either point into the positive or negative $z$ direction, depending on whether $k^{\prime}$ is positive or negative. Energy $E^{\prime}$ and $z$ component of the momentum $k^{\prime}$ are given as follows,

$$
E=\frac{m}{\sqrt{1-u^{2}}}, k=\frac{m u}{\sqrt{1-u^{2}}}, E^{\prime}=\frac{m(1-u v)}{\sqrt{1-v^{2}} \sqrt{1-u^{2}}}, k^{\prime}=\frac{m(u-v)}{\sqrt{1-v^{2}} \sqrt{1-u^{2}}} .
$$

The Lorentz-boosted $k^{\prime}$ is negative for $v>u$, as it should. Let us now investigate the helicity $\sigma^{\prime}$ of the Lorentztransformed spinor. We observe that, according to Eq. (5), setting $\theta=0$ and $\theta=\pi$,

$$
\begin{gathered}
a_{+}\left(|\vec{k}| \hat{e}_{z}\right)=\left(\begin{array}{c}
1 \\
0
\end{array}\right), \quad a_{-}\left(|\vec{k}| \hat{e}_{z}\right)=\left(\begin{array}{l}
0 \\
1
\end{array}\right), \\
a_{+}\left(-|\vec{k}| \hat{e}_{z}\right)=\left(\begin{array}{c}
0 \\
1
\end{array}\right), \quad a_{-}\left(-|\vec{k}| \hat{e}_{z}\right)=\left(\begin{array}{l}
1 \\
0
\end{array}\right) .
\end{gathered}
$$

Here, we have chosen the constant, unobservable phase $\exp ( \pm \mathrm{i} \varphi)$ of the helicity spinor equal so that the nonvanishing entry is equal to unity in each case. The helicity flip in the spinor wave function thus finds a natural mathematical correspondence: Once the $z$ component of the vector $\vec{k}$ flips, the interpretation of the fundamental helicity spinor also flips. Acting with the spinor transformation $S(\Lambda)$ onto the bispinor, it is then possible to verify, after some algebraic manipulation, that the bispinors given in Eq. (3) transform as follows,

$$
\begin{aligned}
& u_{+}(\vec{k}) \rightarrow S(\Lambda) u_{+}(\vec{k})=u_{+}\left(\vec{k}^{\prime}\right)=\left(\begin{array}{c}
\sqrt{\frac{E^{\prime}+m}{2 m}} a_{+}\left(\vec{k}^{\prime}\right) \\
\sqrt{\frac{E^{\prime}-m}{2 m}} a_{+}\left(\vec{k}^{\prime}\right)
\end{array}\right), \quad v<u, \\
& u_{+}(\vec{k}) \rightarrow S(\Lambda) u_{+}(\vec{k})=u_{-}\left(\vec{k}^{\prime}\right)=\left(\begin{array}{c}
\sqrt{\frac{E^{\prime}+m}{2 m}} a_{-}\left(\vec{k}^{\prime}\right) \\
-\sqrt{\frac{E^{\prime}-m}{2 m}} a_{-}\left(\vec{k}^{\prime}\right)
\end{array}\right), \quad v>u, \\
& u_{-}(\vec{k}) \rightarrow S(\Lambda) u_{-}(\vec{k})=u_{-}\left(\vec{k}^{\prime}\right)=\left(\begin{array}{c}
\sqrt{\frac{E^{\prime}+m}{2 m}} a_{-}\left(\vec{k}^{\prime}\right) \\
-\sqrt{\frac{E^{\prime}-m}{2 m}} a_{-}\left(\vec{k}^{\prime}\right)
\end{array}\right), \quad v<u, \\
& u_{-}(\vec{k}) \rightarrow S(\Lambda) u_{-}(\vec{k})=u_{+}\left(\vec{k}^{\prime}\right)=\left(\begin{array}{c}
\sqrt{\frac{E^{\prime}+m}{2 m}} a_{+}\left(\vec{k}^{\prime}\right) \\
\sqrt{\frac{E^{\prime}-m}{2 m}} a_{+}\left(\vec{k}^{\prime}\right)
\end{array}\right), \quad v>u .
\end{aligned}
$$

These equations explicitly verify the presence of the helicity flip, within the bispinor representation of the Lorentz group. Our analysis is in agreement with the brief discussion presented in the text following Eq. (2.331) on p. 46 of Ref. [2]. Helicity flips upon a transformation into a rotating frame of reference are discussed in A. For the two 
helicities $\sigma= \pm$, and the time-like component $\mu=0$, defining ${J^{\prime}}_{\sigma} \equiv{J_{\sigma}^{\prime}}_{\sigma}^{\mu=0}$, we obtain from the Lorentz boost,

$$
\begin{gathered}
J^{\prime}= \begin{cases}\frac{E^{\prime}}{m}\left(1+\sqrt{1-\frac{m^{2}}{E^{\prime 2}}}\right), & v<u, \\
\frac{E^{\prime}}{m}\left(1-\sqrt{1-\frac{m^{2}}{E^{\prime 2}}}\right), & v>u,\end{cases} \\
J_{+}^{\prime}= \begin{cases}\frac{E^{\prime}}{m}\left(1-\sqrt{1-\frac{m^{2}}{E^{2}}}\right), & v<u, \\
\frac{E^{\prime}}{m}\left(1+\sqrt{1-\frac{m^{2}}{E^{\prime 2}}}\right), & v>u .\end{cases}
\end{gathered}
$$

A discussion is in order. The functional form of the Lorentz-transformed amplitudes $J^{\prime}$ - and $J^{\prime}+$ changes at $v=u$, which in turn corresponds to the velocity necessary to overtake the neutrino. The $z$ component of the momentum changes sign at this velocity, and this is manifest in Eq. (16), where the change in the helicity quantum number is manifest. We recall that $\vec{k}^{\prime}=k^{\prime} \hat{e}_{z}$, where $k^{\prime}$ is given in Eq. (14); it changes direction at $v=u$. Indeed, the neutrino is at rest in the moving frame for $v=u$.

The asymptotics of $J^{\prime}{ }_{-}$for $v<u$, for large energy $E^{\prime}$, go as $J^{\prime}{ }_{-} \rightarrow 2 E^{\prime} / m$, in agreement with the left-handed (negative) helicity of the "active" neutrino, while the helicity flip at $v=u$ implies that $J^{\prime}{ }_{-} \rightarrow m /\left(2 E^{\prime}\right)$ for $v>u$, for large energy $E^{\prime}$. In the moving frame, the overtaken neutrino thus becomes gradually sterile, and the change in the functional form in Eq. (17) is consistent with a smooth behaviour of the current amplitude near the point where the neutrino actually is being passed $(v=u)$. The suppression factor upon comparing the two asymptotic forms thus reads as $m^{2} /\left(4 E^{\prime 2}\right)$, where $E^{\prime}$ is given according to Eq. (14) (see also Fig. 11).

Conversely, the asymptotics of $J^{\prime}+$ for $v<u$, for large energy $E^{\prime}$, go as $J^{\prime}+m /\left(2 E^{\prime}\right)$ and are thus suppressed, in agreement with the right-handed (negative) helicity of the "active" neutrino, while the helicity flip at $v=u$ implies that $J^{\prime}{ }_{+} \rightarrow 2 E^{\prime} / m$ for $v>u$, for large energy $E^{\prime}$. In the moving frame, the overtaken neutrino is "de-sterilized". Despite this parametric suppression at high energies, the right-handed Dirac neutrinos are thus not completely sterile. This consideration quantifies and illustrates the "helicity reversal question" which underlies the considerations of Refs. 24 26], and Fig. 2 clearly demonstrates how the helicity reversal and the concomitant gradual suppression of the weak interaction occurs as the velocity of the reference frame $v$ approaches the speed $u$ of the neutrino.

\section{MAJORANA PARTICLES AND HELICITY FLIP}

Recently, Majorana-particle-like excitations have been observed in solid-state physics [29]. Also, a quantum propagation algorithm [30] for Majorana particle wave packets has been devised, which takes the particle-antiparticle symmetry of the Majorana wave function into account. These questions are nontrivial because the Majorana particle formally follows the Dirac equation, but with the additional constraint that the wave function is charge-conjugation invariant, i.e. it fulfills the condition $\psi^{\mathcal{C}}=C \bar{\psi}^{\mathrm{T}}$ with $C=\mathrm{i} \gamma^{2} \gamma^{0}$ (in the Dirac representation which we use here).

At variance with Ref. [1], we here consider a space-time metric has the signature $(+,-,-,-)$ so that $k \cdot x=E t-\vec{k} \cdot \vec{r}$. The Majorana fermion is described by the field operator [see Eq. (3.25) on p. 47 of Ref. 1]],

$$
\hat{\psi}(x)=\sum_{\vec{k}, \sigma} \sqrt{\frac{m}{E V}}\left[f_{\sigma}(\vec{k}) u_{\sigma}(\vec{k}) \mathrm{e}^{-\mathrm{i} k \cdot x}+\lambda f_{-\sigma}^{+}(\vec{k}) v_{-\sigma}(\vec{k}) \mathrm{e}^{\mathrm{i} k \cdot x}\right]
$$

where the operator $f_{\sigma}(\vec{k})$ annihilates a Majorana fermion, whereas $f_{\sigma}^{+}(\vec{k})$ creates one. The peculiar $-\sigma$ subscript in the antiparticle contribution to $\hat{\psi}(x)$ stems from the fact that in our conventions, $\sigma$ is a quantum number which is equal to the helicity for particles, whereas it is equal to the negative of the helicity for antiparticles [see the text following Eq. (4) and the discussion in C especially Eq. (C2)]. The creation phase factor $\lambda=\exp (\mathrm{i} \varphi)$ has unit modulus [1]. Up to a phase factor, the Majorana field operator is equal to its own charge conjugate $\mathcal{C} \hat{\psi}(x) \mathcal{C}^{-1}$ [see Eq. (3.29) on p. 51 of Ref. [1]]. This form of the field operator ensures that the time-ordered vacuum expectation value reproduces the Feynman form i/ $(k-m+\mathrm{i} \epsilon)$ in momentum space (see p. 67 of Ref. [1]).

Because a Majorana fermion is equal to its own antiparticle, the vector and axial vector currents corresponding to single-particle state of the Majorana inevitably acquire contributions from the anti-particle solutions. The correspondence is as follows. We consider the matrix elements $\left\langle\nu_{f}\left|\hat{\bar{\psi}} \gamma^{\mu} \hat{\psi}\right| \nu_{i}\right\rangle$ and $\left\langle\nu_{f}\left|\hat{\bar{\psi}} \gamma^{\mu} \gamma^{5} \hat{\psi}\right| \nu_{i}\right\rangle$ where $\hat{\psi}$ is the 


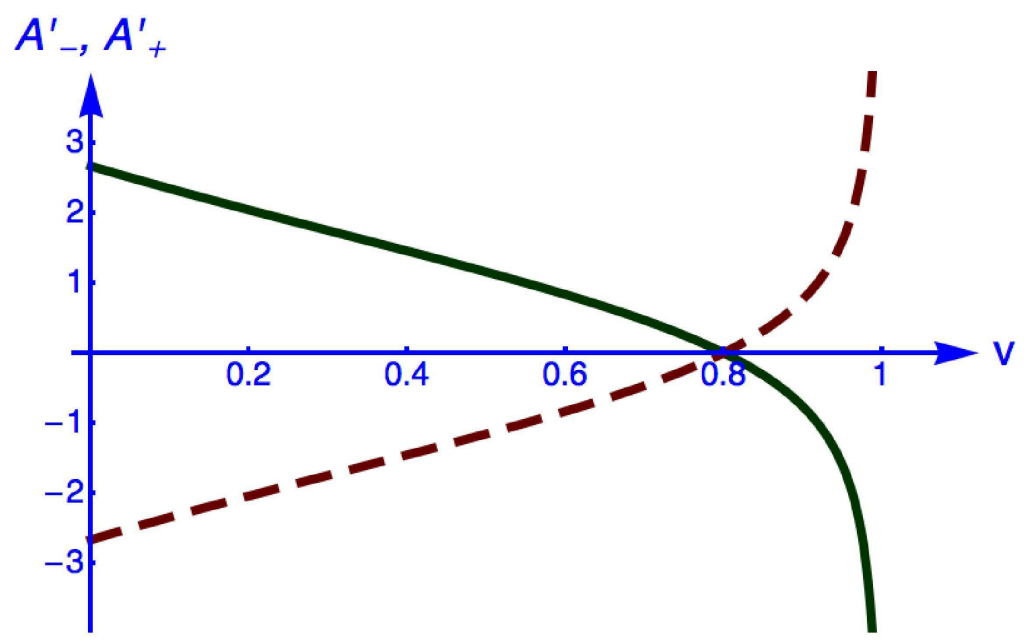

FIG. 3: The axial-vector matrix element $A_{\sigma}^{\prime}$ for Majorana neutrinos replaces the current $J_{\sigma}^{\prime}$ plotted for Dirac particles in Fig. 2 (the solid curve is for $A_{-}^{\prime}$, the dashed one for $A_{+}^{\prime}$ ). According to Eq. (27), the helicity of the Majorana neutrino flips (we again use a value of $u=0.8$ for the velocity, the neutrino is overtaken for $v>u$ ). However, there is no sterilization as one boosts past the Majorana neutrino.

Majorana field operator and $\left|\nu_{i}\right\rangle=f_{\sigma}^{+}(\vec{k})|0\rangle$ is a one-particle state lifted from the vacuum (the subscript $i$ and $f$ denote initial and final states). According to the derivation presented on pp. 61-62 of Ref. [1], one can show that

$$
\begin{aligned}
\left\langle\nu_{f}\left|\hat{\bar{\psi}} \gamma^{\mu} \hat{\psi}\right| \nu_{i}\right\rangle & \propto \bar{u}_{f} \gamma^{\mu} u_{i}-\bar{v}_{i} \gamma^{\mu} v_{f} \equiv V^{\mu}, \\
\left\langle\nu_{f}\left|\hat{\bar{\psi}} \gamma^{\mu} \gamma^{5} \hat{\psi}\right| \nu_{i}\right\rangle & \propto-\left(\bar{u}_{f} \gamma^{\mu} \gamma^{5} u_{i}-\bar{v}_{i} \gamma^{\mu} \gamma^{5} v_{f}\right) \equiv A^{\mu},
\end{aligned}
$$

We here define the vector current and axial vector current matrix elements $V^{\mu}$ and $A^{\mu}$, respectively. The negative prefactor in the definition of $A^{\mu}$ will find an immediate explanation. For forward scattering, the matrix elements corresponding to $J_{\sigma}^{\mu}$ defined in Eq. (10) are thus as follows,

$$
\begin{aligned}
V_{\sigma}^{\mu} & =\bar{u}_{\sigma}(\vec{k}) \gamma^{\mu} u_{\sigma}(\vec{k})-\bar{v}_{-\sigma}(\vec{k}) \gamma^{\mu} v_{-\sigma}(\vec{k}), \\
A_{\sigma}^{\mu} & =-\left(\bar{u}_{\sigma}(\vec{k}) \gamma^{\mu} \gamma^{5} u_{\sigma}(\vec{k})-\bar{v}_{-\sigma}(\vec{k}) \gamma^{\mu} \gamma^{5} v_{-\sigma}(\vec{k})\right) .
\end{aligned}
$$

The charge conjugation matrix $C=\mathrm{i} \gamma^{2} \gamma^{0}$ has the properties,

$$
\begin{aligned}
C & =\mathrm{i} \gamma^{2} \gamma^{0}, \quad C^{2}=-\mathbf{1}_{4 \times 4}, \quad C^{-1}=-C, \\
C^{-1} \gamma^{\mu} C & =-\left(\gamma^{\mu}\right)^{\mathrm{T}}, \quad C^{-1} \gamma^{5} C=\left(\gamma^{5}\right)^{\mathrm{T}}, \\
v_{-\sigma}(\vec{k}) & =C \bar{u}_{\sigma}^{\mathrm{T}}(\vec{k}), \quad \bar{v}_{-\sigma}(\vec{k})=-u_{\sigma}^{\mathrm{T}}(\vec{k}) C^{-1},
\end{aligned}
$$

where $\mathbf{1}_{4 \times 4}$ is the four-dimensional unit matrix. Hence, for the vector current, we have

$$
\bar{v}_{-\sigma}(\vec{k}) \gamma^{\mu} v_{-\sigma}(\vec{k})=-u_{\sigma}^{\mathrm{T}}(\vec{k}) C^{-1} \gamma^{\mu} C \bar{u}_{\sigma}^{\mathrm{T}}(\vec{k})=\bar{u}_{\sigma}(\vec{k}) \gamma^{\mu} u_{\sigma}(\vec{k}),
$$

whereas for the axial vector current,

$$
\begin{aligned}
\bar{v}_{-\sigma}(\vec{k}) \gamma^{\mu} \gamma^{5} v_{-\sigma}(\vec{k}) & =-u_{\sigma}^{\mathrm{T}}(\vec{k}) C^{-1} \gamma^{\mu} C C^{-1} \gamma^{5} C \bar{u}_{\sigma}(\vec{k}) \\
& =-u_{\sigma}^{\mathrm{T}}(\vec{k})\left(-\gamma^{\mu}\right)^{\mathrm{T}}\left(\gamma^{5}\right)^{\mathrm{T}} \bar{u}_{\sigma}(\vec{k})=u_{\sigma}^{\mathrm{T}}(\vec{k})\left(\gamma^{5} \gamma^{\mu}\right)^{\mathrm{T}} \bar{u}_{\sigma}^{\mathrm{T}}(\vec{k}) \\
& =-u_{\sigma}^{\mathrm{T}}(\vec{k})\left(\gamma^{\mu} \gamma^{5}\right)^{\mathrm{T}} \bar{u}_{\sigma}^{\mathrm{T}}(\vec{k})=-\bar{u}_{\sigma}(\vec{k}) \gamma^{\mu} \gamma^{5} u_{\sigma}(\vec{k}) .
\end{aligned}
$$

So, we have

$$
V_{\sigma}^{\mu}=0, \quad A_{\sigma}^{\mu}=-2 \bar{u}_{\sigma}(\vec{k}) \gamma^{\mu} \gamma^{5} u_{\sigma}(\vec{k}),
$$


a result which confirms the common wisdom that Majorana particles have no vector current (see p. 60 of Ref. [1]). The $V$ - $A$ coupling for Majorana particles is exclusively carried by the axial component. The prefactor in $A_{\sigma}^{\mu}$ is chosen so that, in the correspondence of Eqs. (10) and (25),

$$
J_{\sigma}^{\mu} \quad(\text { for Dirac neutrinos }) \Leftrightarrow A_{\sigma}^{\mu} \quad \text { (for Majorana neutrinos). }
$$

The amplitudes $J_{\sigma}^{\mu} \Leftrightarrow A_{\sigma}^{\mu}$ are matched in the high-energy limit where the helicity eigenstates approximate the chirality eigenstates and are "almost" eigenstates of $\gamma^{5}$. In analogy to Eq. (17), we define $A_{\sigma}^{\prime}=A_{\sigma}^{\prime \mu=0}$ for the time-like component. The following results are easily obtained for the Lorentz-transformed axial Majorana currents,

$$
\begin{aligned}
& A^{\prime}{ }_{-}= \begin{cases}\frac{2 E^{\prime}}{m} \sqrt{1-\frac{m^{2}}{E^{\prime 2}}}, & v<u, \\
-\frac{2 E^{\prime}}{m} \sqrt{1-\frac{m^{2}}{E^{\prime 2}}}, & v>u,\end{cases} \\
& A^{\prime}= \begin{cases}-\frac{2 E^{\prime}}{m} \sqrt{1-\frac{m^{2}}{E^{\prime 2}}}, & v>u, \\
\frac{2 E^{\prime}}{m} \sqrt{1-\frac{m^{2}}{E^{\prime 2}}}, & v>u .\end{cases}
\end{aligned}
$$

These results confirm the Dirac-Majorana confusion theorem 31$] 33$ : For $E^{\prime} \gg m$, we have $J_{\sigma}^{\prime} \approx A_{\sigma}^{\prime} \approx 2 E^{\prime} / m$, but this holds only for Lorentz transformations into frames which do not "overtake" the neutrino $(v<u)$. As we "overtake" the Majorana neutrino, the left-handed Majorana neutrino transforms into a right-handed Majorana neutrino, which however, because the Majorana is its own anti-particle, needs to interact as if it were a right-handed Majorana anti-neutrino, hence it is not sterile. This is illustrated in Fig. 3. Majorana neutrinos are not sterilized by overtaking them in a gedanken experiment on a highway. To put the conclusion into perspective, a left-handed neutrino incident on a target at rest in the frame in which it is born will generate leptons, but when one boosts past it and the helicity flips, if it is a Majorana particle, then an interaction with a target at rest in the boosted frame will generate antileptons. This is consistent with the lepton-number nonconservation induced into the Standard Model by Majorana neutrinos (an illustrative discussion is found in Sec. 6.1.1 on pp. 184-185 of Ref. [2]).

\section{GENERALIZED DIRAC EQUATION AND HELICITY SUPPRESSION}

In order to start the discussion of generalized Dirac equations with exotic dispersion relations, we first approach the light cone by considering massless Dirac fermions, which are always in a helicity eigenstate, if they are in an energy eigenstate. The massless Dirac equation and its Hamiltonian $H_{0}$ read, quite simply, i $\gamma^{\mu} \partial_{\mu} \psi(x)=0$, and $H_{0}=\vec{\alpha} \cdot \vec{p}$, respectively, where we again use the standard Dirac representation, and the $\vec{\sigma}$ are the $2 \times 2$ Pauli matrices. The massless Dirac Hamiltonian is a $4 \times 4$ matrix and it is simultaneously Hermitian as well as pseudo-Hermitian or " $\gamma^{5}$-Hermitian" [34 43],

$$
H_{0}=H_{0}^{+}, \quad H_{0}=\gamma^{5} H_{0}^{+} \gamma^{5} .
$$

One chooses a plane-wave ansatz of the form $\psi(x)=u_{\sigma}(\vec{k}) \exp (-\mathrm{i} k \cdot x)$ for particles and $\psi(x)=v_{\sigma}(\vec{k}) \exp (\mathrm{i} k \cdot x)$ for antiparticles, where $k \cdot x=|\vec{k}| t-\vec{k} \cdot \vec{r}$. According to Sec. 2.4.3 of Ref, [44], the canonical choice for the phases of the fundamental eigenspinors is as follows,

$$
\begin{aligned}
& u_{+}(\vec{k})=\frac{1}{\sqrt{2}}\left(\begin{array}{c}
a_{+}(\vec{k}) \\
a_{+}(\vec{k})
\end{array}\right), \quad u_{-}(\vec{k})=\frac{1}{\sqrt{2}}\left(\begin{array}{c}
a_{-}(\vec{k}) \\
-a_{-}(\vec{k})
\end{array}\right), \\
& v_{+}(\vec{k})=\frac{1}{\sqrt{2}}\left(\begin{array}{c}
-a_{+}(\vec{k}) \\
-a_{+}(\vec{k})
\end{array}\right), \quad v_{-}(\vec{k})=\frac{1}{\sqrt{2}}\left(\begin{array}{c}
-a_{-}(\vec{k}) \\
a_{-}(\vec{k})
\end{array}\right) .
\end{aligned}
$$

The zero-mass solutions simultaneously fulfill the following projector identities,

$$
\begin{gathered}
\sum_{\sigma} 2|\vec{k}| u_{\sigma}(\vec{k}) \otimes \bar{u}_{\sigma}(\vec{k})=\sum_{\sigma} 2|\vec{k}| v_{\sigma}(\vec{k}) \otimes \bar{v}_{\sigma}(\vec{k})=\not k \\
\sum_{\sigma} 2|\vec{k}|(-\sigma) u_{\sigma}(\vec{k}) \otimes \bar{u}_{\sigma}(\vec{k}) \gamma^{5}=\sum_{\sigma} 2|\vec{k}|(-\sigma) v_{\sigma}(\vec{k}) \otimes \bar{v}_{\sigma}(\vec{k}) \gamma^{5}=\not k
\end{gathered}
$$


as one can check by an explicit calculation. We note, in particular, that it is impossible to "choose a covariant normalization" in the massless case; one always has $\bar{u}_{\sigma}(\vec{k}) u_{\sigma}(\vec{k})=u_{\sigma}^{+}(\vec{k}) \gamma^{0} u_{\sigma}(\vec{k})=0$ and $\bar{v}_{\sigma}(\vec{k}) v_{\sigma}(\vec{k})=0$ for the massless spinors given in Eq. (29), hence, the prefactors $|\vec{k}|$ in Eq. (30).

The tachyonic generalized Dirac equation reads as

$$
\left(\mathrm{i} \gamma^{\mu} \partial_{\mu}-\gamma^{5} m\right) \psi(x)=0
$$

with a Lagrangian density $\mathcal{L}=\bar{\psi}(x) \gamma^{5}\left(\mathrm{i} \gamma^{\mu} \partial_{\mu}-\mathrm{i} m\right) \psi(x)$. The corresponding Hamiltonian $[7-13]$ is pseudo-Hermitian or " $\gamma^{5}$-Hermitian",

$$
H=\vec{\alpha} \cdot \vec{p}+\beta \gamma^{5} m, \quad H=\gamma^{5} H^{+} \gamma^{5} .
$$

The concept of pseudo-Hermiticity has been established as a viable generalization of Hermiticity for quantum systems [34 42], with pseudo-Hermitian Hamiltonians describing systems where the absorptive ("gain") and dissipative ("loss") terms are in equilibrium, and the resulting eigenenergy is real rather than complex [45]. Such systems are physically realized in so-called $\mathcal{P} \mathcal{T}$-symmetric waveguides [46] and optical lattices [47]. The application to a superluminal spin-1/2 particle constitutes a candidate theory for a more fundamental application of the concept of pseudo-Hermiticity, whose phenomenological relevance remains to be tested experimentally.

The fundamental positive-energy and negative-energy eigenstates of the tachyonic Dirac equation in the helicity basis are given as $\psi(x)=u_{\sigma}(\vec{k}) \mathrm{e}^{\mathrm{i} k \cdot x}$ and $\psi(x)=v_{\sigma}(\vec{k}) \mathrm{e}^{\mathrm{i} k \cdot x}$ with with $k \cdot x=E t-\vec{k} \cdot \vec{r}$ and $E=\sqrt{\vec{k}^{2}-m^{2}}$. The fundamental eigenspinors are obtained from Eqs. (3) and (4) by the simple (formal) substitution $E \rightarrow|\vec{k}|$ (see Ref. [13]),

$$
u_{+}(\vec{k})=\left(\begin{array}{c}
\sqrt{\frac{|\vec{k}|+m}{2 m}} a_{+}(\vec{k}) \\
\sqrt{\frac{|\vec{k}|-m}{2 m}} a_{+}(\vec{k})
\end{array}\right), \quad u_{-}(\vec{k})=\left(\begin{array}{c}
\sqrt{\frac{|\vec{k}|+m}{2 m}} a_{-}(\vec{k}) \\
-\sqrt{\frac{|\vec{k}|-m}{2 m}} a_{-}(\vec{k})
\end{array}\right),
$$

with the normalization $\bar{u}_{\sigma}(\vec{k}) u_{\sigma}(\vec{k})=\sigma$, and

$$
v_{+}(\vec{k})=\left(\begin{array}{l}
-\sqrt{\frac{|\vec{k}|-m}{2 m}} a_{+}(\vec{k}) \\
-\sqrt{\frac{|\vec{k}|+m}{2 m}} a_{+}(\vec{k})
\end{array}\right), \quad v_{-}(\vec{k})=\left(\begin{array}{c}
-\sqrt{\frac{|\vec{k}|-m}{2 m}} a_{-}(\vec{k}) \\
\sqrt{\frac{|\vec{k}|+m}{2 m}} a_{-}(\vec{k})
\end{array}\right),
$$

with the normalization $\bar{v}_{\sigma}(\vec{k}) v_{\sigma}(\vec{k})=-\sigma$. The projector sums reads as follows,

$$
\sum_{\sigma}(-\sigma) u_{\sigma}(\vec{k}) \otimes \bar{u}_{\sigma}(\vec{k}) \gamma^{5}=\frac{\not k-\gamma^{5} m}{2 m}, \quad \sum_{\sigma}(-\sigma) v_{\sigma}(\vec{k}) \otimes \bar{v}_{\sigma}(\vec{k}) \gamma^{5}=\frac{\not k+\gamma^{5} m}{2 m} .
$$

We recall that $\sigma$ is a "good" quantum number and characterizes the helicity, not chirality. Indeed, $\sigma$ is equal to the helicity for positive-energy states, and equal to minus the helicity for negative-energy states. In the massless limit [13], the "second" projector sum (30b) is recovered.

One might wonder about the "mysterious" factor $(-\sigma)$ in the sum rules. If we postulate that the time-ordered product of field operators should give a propagator whose Fourier transformation is the inverse of the Hamiltonian, then we have to sum over the eigenspinors, as is done in any derivation of a Green function, and obtain the positiveenergy and negative-energy projectors over the eigenspinors as a result of the spin sums [13]. So, we have to postulate that an expression of the form

$$
\sum_{\operatorname{spin}}(\text { prefactor }) \times(\text { tensor product of states }) \times(\text { scalar or pseudo-scalar matrix })
$$

must be equal to a positive-energy or negative-energy projector. Here, the prefactor can only come from the fundamental anticommutator of the field operator, which in turn can only involve the "good" quantum numbers. We postulate a relation of the form

$$
\left\{b_{\sigma}(k), b_{\rho}^{+}\left(k^{\prime}\right)\right\}=f(\sigma, \vec{k})(2 \pi)^{3} \frac{E}{m} \delta^{3}\left(\vec{k}-\vec{k}^{\prime}\right) \delta_{\sigma \rho},
$$


where the $b$ and $b^{+}$annihilate and create particles of the respective quantum numbers, and corresponding relations for the creators and annihilators of antiparticles. The "prefactor" in Eq. (36) has to be a function of "good" quantum numbers [11 13 ] and therefore of the functional form $f(\sigma, \vec{k})$,

$$
\text { (prefactor) }=f(\sigma, \vec{k}) \text {, }
$$

where $f(\sigma, \vec{k})$ enters Eq. (37). For the further derivation of the tachyonic propagator, one simply generalizes the derivation that leads from Eq. (3.169) to Eq. (3.170) in the standard textbook [44], as it has been described in Ref. [13]. In turn, an inspection of Eqs. (6) and (35) shows that for the choices

$$
f(\sigma, \vec{k})=1 \quad(\text { tardyonic choice }), \quad f(\sigma, \vec{k})=-\sigma \quad(\text { tachyonic choice })
$$

the sum rules (6) and (35) are fulfilled, and the propagator may be calculated [13]. If we accept the anticommutator relation (37), then the right-handed helicity state acquires negative norm. This is seen as follows,

$$
\left\langle 1_{k, \sigma} \mid 1_{k, \sigma}\right\rangle=\left\langle 0\left|b_{\sigma}(k) b_{\sigma}^{+}(k)\right| 0\right\rangle=\left\langle 0\left|\left\{b_{\sigma}(k), b_{\sigma}^{+}(k)\right\}\right| 0\right\rangle=(-\sigma) V \frac{E}{m},
$$

where $V=(2 \pi)^{3} \delta^{3}(\overrightarrow{0})$ is the normalization volume in coordinate space. The norm $\left\langle 1_{k, \sigma} \mid 1_{k, \sigma}\right\rangle$ is negative for $\sigma=1$. Therefore, right-handed particle states are excluded from the spectrum by a Gupta-Bleuler condition [11, 13]. This is analogous to virtual photons which can be scalar or longitudinal, but physical photons must be transverse (see also B). The helicity reversal question discussed in Sec. II does not need to be addressed for (even infinitesimally) superluminal neutrinos because it is impossible to overtake them, starting from rest, by the laws of special relativity.

\section{CONCLUSIONS}

We have presented an overview of helicity suppression mechanisms for the "wrong" neutrino helicity states, under the assumption that the neutrino mass eigenstates are either described by the standard Dirac equation (see Sec. II), or by the tachyonic Dirac equation (see Sec. IV]. The case of a Majorana neutrino is treated in Sec. III] For a Dirac neutrino, incomplete sterilization occurs if one overtakes a left-handed neutrino while speeding on a highway without speed limits. The same mechanism predicts gradual de-sterilization upon overtaking right-handed Dirac neutrinos. This phenomenon is graphically represented in Fig. 2, The weak interactions of the "wrong" helicity states, for a standard Dirac neutrino, are suppressed by a power of $m / E$, where $m$ is the neutrino mass and $E$ is the energy scale. In addition, one should point out that the (almost) sterile "left-handed turned right-handed" (after the passing maneuver) Dirac neutrinos still have the same mass as before the overtaking: They represent not-quite-sterile, light, right-handed states of the fermion field. Perhaps even more counter-intuitive (but consistently described by a spinor Lorentz transformation) is the gradual de-sterilization of a bunch of right-handed neutrinos as one passes them on a highway (see also Fig. 2).

By contrast, for Majorana neutrinos, no sterilization occurs (see Sec. III). The antiparticle-component of the axial vector current, which is always present for a Majorana particle, leads to a "built-in" transformation of the Majorana neutrino into its own antiparticle upon overtaking it on a highway, and thus, to de-sterilization upon transformation to a very fast reference frame (see Fig. 3). Also, we recall that for Majorana neutrinos, one particular set of helicity states (right-handed neutrino states whose antiparticles are left-handed anti-neutrinos) acquire a very large mass within the seesaw mechanism (see p. 100 of Ref. [3] ), leading to a much more effective suppression involving a power of $m / \Lambda_{\mathrm{GUT}}$.

One might refer to the underlying questions, which have been discussed in Refs. [24 26], as the "helicity reversal paradox", the "autobahn helicity paradox", or, with reference to Ref. [23], the "rabbit paradox". One might argue that the helicity reversal of Sec. II is described by a Lorentz transformation and does not constitutes a paradox. The same argument, though, would otherwise apply to the "twin paradox" of time dilation which can be "resolved" by pointing to the asymmetry of the problem, in view of the necessary acceleration of the space craft as the "younger" twin reverses the course. Also, the "Ehrenfest paradox" (see Ref. [48]) which applies to the fast rotation speeds exceeding the speed of light in revolving Lorentz frames, for points sufficiently displaced from the rotation axis, can be resolved by carefully analyzing the physical interpretation of the coordinates [49, 50]. However, it appears to be customary to refer to an intriguing, counter-intuitive (relativistic) phenomenon as a "paradox", and this is why we would advocate the designation of a somewhat paradoxical status to "question \#76" (see Refs. 25, 26]) connected with the helicity reversal upon a Lorentz boost. In any case, the question becomes a true paradox if we additionally assume that the apparent absence of right-handed neutrinos in nature is due to some more fundamental reason such as the exclusion from the physical spectrum by a Gupta-Bleuler condition [13] rather than practical difficulties incurred 
in the detection of the right-handed states. In Sec. 3 of Ref. [14], the problem is solved by simply projecting the neutrino field, with general Dirac and Majorana mass terms, by projecting the entire neutrino field onto its lefthanded component. We might add that the "almost" sterile, light, right-handed, overtaken Dirac neutrinos cannot be interpreted as anti-neutrinos because the energy of the left-handed Dirac neutrinos does not change sign upon a Lorentz boost given in Eq. (12).

A superluminal neutrino, described by a generalized Dirac equation with a pseudo-scalar mass term (see Sec. III), has the potential to address a few physical questions connected with neutrinos. The "wrong helicity states" are completely suppressed from the physical neutrino fields due to Gupta-Bleuler condition, and this remains true for infinitesimally superluminal neutrinos with a very small tachyonic mass, because superluminal particles always remain superluminal upon Lorentz transformation. One cannot overtake an ever-so-slightly superluminal neutrino. This follows from the Lorentz transformation, by which superluminal particles always remain superluminal under a transformation between subluminal reference frames. The tachyonic Dirac equation allows for plane-wave solutions, so that essentially nothing has to be altered in electroweak theory if we accept the fact that subluminal and superluminal particles may couple through Lorentz-invariant interactions. Perhaps, one might investigate, in the future, a scenario where neutrinos are superluminal "but not as superluminal as recent false and retracted experimental claims" would otherwise suggest.

Experimental determinations of the neutrino mass square have typically resulted in negative expectation values, yet, compatible with zero within experimental error bounds. Also, experiments on the neutrino propagation velocity have typically yielded results compatible with $v=c$ within error. Quoting Hilbert, we "must know" and "will know" as the experimental accuracy of time-of-flight and neutrinoless beta decay experiments improves.

\section{Acknowledgements}

The authors acknowledge helpful conversations with R. J. Hill, W. Rodejohann, T. N. Moentmann and I. Nándori. Furthermore, insightful remarks from an anonymous referee are acknowledged. This paper is in part based in part on discussions held during the First International Conference on Logic and Relativity, Alfréd Rényi Institute of Mathematics, September 2012, Budapest, Hungary. Thus authors wish to acknowledge insightful conversations with Professors István Nemeti and Gergely Székely. The work was completed during the summer months of 2013 when one of the authors (U.D.J.) was primarily working at the ATOMKI institute of the Hungarian Academy of Sciences in Debrecen; kind hospitality is gratefully acknowledged. Support by the National Science Foundation (Grant PHY1068547) and by the National Institute of Standards and Technology (precision measurement grant) is also gratefully acknowledged.

\section{Appendix A: Fast Rotations and Helicity Flip}

In Sec. III, we have calculated the helicity flip of a neutrino, overtaken on a highway. One might think that the same helicity flip might occur in a reference frame which rotates around the propagation direction (assumed to be the $z$ axis) of the neutrino, imagining that the spin of a particle can be viewed as the rotation of a spinning top. Let us first present an intuitive, classical argument suggesting that a helicity flip cannot occur upon transformation to the rotating frame and then supplement this argument by a full quantum calculation, in the spinor formalism.

The classical argument is based on the following observation: Imagine the neutrino as a spinning top with a (classical) angular momentum component $L_{z}=\frac{1}{2} \hbar$. If the mass of the spinning top is concentrated on a ring displaced by a radius $r$ from the central rotation axis, and $m$ is the mass of the spinning classical "neutrino", then the angular momentum is $L_{z}=r m v$, where $v$ is the rotation speed of the mass distribution. The required speed is equal to the speed of light at a radius governed by the equation

$$
L_{z}=\frac{1}{2} \hbar=r m v=r m c, \quad r=\frac{\hbar}{2 m c}=\frac{\lambda}{2},
$$

where $\lambda$ is the reduced Compton wavelength. One cannot localize a quantum particle better than its Compton wavelength, and therefore the quantum-classical analogue of a spinning top makes sense provided we assume that the particle's mass distribution is displaced from the central rotation axis by a distance commensurate with the particle's Compton wavelength. Conversely, only an "outside" observer could potentially see the neutrino spinning in the opposite direction and reverse its helicity; if we are "inside" the neutrino's Compton wavelength then the concept of a classical spinning top becomes meaningless. Equation (A1) teaches us that if we are to overtake the rotating spinning top by spinning around the axis of rotation faster than the neutrino spin does, and revert its helicity, then we have to rotate about the symmetry axis with a velocity faster than the speed of light. This is impossible for a classical observer starting from rest, according to special relativity and therefore, the classical consideration strongly 
suggest that helicity reversal in a rotating frame is impossible. (With reference to Sec. III of this article, we should reconfirm that, by contrast, tachyons are never at rest, and remain superluminal in reference frame connected by a proper orthochronous Lorentz transformation [51 55].)

Let us supplement this classical consideration by a full quantum calculation. Setting aside possible complications due to rotation speeds faster than $c$ in rotating frames (otherwise known as the Ehrenfest paradox [48 50]), we write down the Lorentz and spinor transformation for the transformation into the rotating frame in full analogy to Eq. (12) as follows,

$$
\begin{aligned}
\Lambda_{\nu}^{\mu} & =\left(\begin{array}{cccc}
1 & 0 & 0 & 0 \\
0 & \cos \left(\omega_{0} t\right) & \sin \left(\omega_{0} t\right) & 0 \\
0 & -\sin \left(\omega_{0} t\right) & \cos \left(\omega_{0} t\right) & 0 \\
0 & 0 & 0 & 1
\end{array}\right), \\
S(\Lambda) & =\left(\begin{array}{cccc}
\exp \left(\frac{\mathrm{i} \omega_{0} t}{2}\right) & 0 & 0 & 0 \\
0 & \exp \left(-\frac{\mathrm{i} \omega_{0} t}{2}\right) & 0 & 0 \\
0 & 0 & \exp \left(\frac{\mathrm{i} \omega_{0} t}{2}\right) & 0 \\
0 & 0 & 0 & \exp \left(-\frac{\mathrm{i} \omega_{0} t}{2}\right)
\end{array}\right) .
\end{aligned}
$$

Here, $\omega_{0}$ is the angular frequency of the rotation. We perform a passive rotation, i.e., the transformed coordinates correspond to the rotating frame, which rotates about the $z$ axis in the counter-clockwise (mathematically positive) direction. The generators for the rotation, in the sense of Eq. (13), read as $\omega_{21}=-\omega_{12}=\omega_{0} t=\varphi$, with all other elements vanishing (here, $\varphi$ is the azimuthal rotation angle). The wave vector $\vec{k}=k \hat{e}_{z}$ is invariant under the rotation, and the effect of the rotation into the rotating frame can be illustrated for the positive-helicity state $u_{+}\left(k \hat{e}_{z}\right)$ defined according to Eq. (3) as follows,

$$
\begin{aligned}
& u_{+}\left(k \hat{e}_{z}\right) \mathrm{e}^{\mathrm{i} k z-\mathrm{i} E t}=\left(\begin{array}{c}
\sqrt{\frac{E+m}{2 m}}\left(\begin{array}{l}
1 \\
0
\end{array}\right) \\
\sqrt{\frac{E-m}{2 m}}\left(\begin{array}{l}
1 \\
0
\end{array}\right)
\end{array}\right) \\
& \rightarrow S(\Lambda) u_{+}\left(k \hat{e}_{z}\right) \mathrm{e}^{\mathrm{i} k z-\mathrm{i} E t}=\mathrm{e}^{\mathrm{i} \omega_{0} t / 2}\left(\begin{array}{c}
\sqrt{\frac{E+m}{2 m}}\left(\begin{array}{l}
1 \\
0
\end{array}\right) \\
\sqrt{\frac{E-m}{2 m}}\left(\begin{array}{l}
1 \\
0
\end{array}\right)
\end{array}\right) \mathrm{e}^{\mathrm{i} k z-\mathrm{i} E t} .
\end{aligned}
$$

We here indicate the dependence of the bispinor wave function on the spatio-temporal phase factor explicitly; the expression $k z-E t$ is invariant under the rotation about the $z$ axis. The energy, as measured in the rotating frame, is decreased by an amount $-\omega_{0} t / 2$, by virtue of the definition of the Hamilton operator as $H=\mathrm{i} \partial_{t}$. This is in full analogy to a classical consideration, where the angular frequency of the spinning top (we note the counter-clockwise classical rotation spin for positive helicity) is decreased in the rotating frame. This situation is reversed for negative helicity, where according to Eq. (A2) the time-dependent phase factor is $\exp \left(-i \omega_{0} t / 2\right)$, and the rotation speed is higher in the rotating frame, resulting in an energy increase by $\omega_{0} t / 2$.

An inspection of the transformed wave function in Eq. (A3) shows that the helicity in the rotating frame is not reversed, irrespective of the magnitude of the angular rotation frequency $\omega_{0}$. Namely, one can easily show that the operator that measures the helicity for a particle propagating in the positive $z$ direction is $\vec{\Sigma} \cdot\left(k \hat{e}_{z}\right) / k=\Sigma^{3}$, where

$$
\Sigma^{i}=\left(\begin{array}{cc}
\sigma^{i} & 0 \\
0 & \sigma^{i}
\end{array}\right), \quad i=1,2,3,
$$

is a $4 \times 4$ spin matrix. This finding in turn confirms the classical argument regarding the rotation about the central axis faster than the speed of light, which is impossible for massive observers starting from rest.

Let us also address the question of a conceivable helicity reversal in the transformation into a frame which rotates at an angular frequency described by the vector

$$
\vec{\omega}=\omega_{0} \hat{n}, \quad \hat{n}=\sin \theta_{\omega} \cos \varphi_{\omega} \hat{e}_{x}+\sin \theta_{\omega} \sin \varphi_{\omega} \hat{e}_{y}+\cos \theta_{\omega} \hat{e}_{z} .
$$

Under the rotation with angle $\vec{\varphi}=\vec{\omega} t=\omega_{0} \hat{n} t$, with Lorentz generators

$$
\Lambda_{\mu \nu} \approx \delta_{\mu \nu}+\omega_{\mu \nu}, \quad \omega_{\mu \nu}=\left(\begin{array}{cccc}
0 & 0 & 0 & 0 \\
0 & 0 & \varphi_{z} & -\varphi_{y} \\
0 & -\varphi_{z} & 0 & \varphi_{x} \\
0 & \varphi_{y} & -\varphi_{x} & 1
\end{array}\right)
$$


the initial wave vector $\vec{k}=k \hat{e}_{z}$ transforms into $\vec{k}^{\prime}=k_{x}^{\prime} \hat{e}_{x}+k_{y}^{\prime} \hat{e}_{y}+k_{z}^{\prime} \hat{e}_{z}$ with

$$
\begin{aligned}
& k_{x}^{\prime}=\sin \left(\theta_{\omega}\right)\left(2 \cos \left(\theta_{\omega}\right) \cos \left(\varphi_{\omega}\right) \sin ^{2}\left(\frac{1}{2} \omega_{0} t\right)-\sin \left(\varphi_{\omega}\right) \sin \left(\omega_{0} t\right)\right), \\
& k_{y}^{\prime}=\sin \left(\theta_{\omega}\right)\left(2 \cos \left(\theta_{\omega}\right) \cos \left(\varphi_{\omega}\right) \sin ^{2}\left(\frac{1}{2} \omega_{0} t\right)+\cos \left(\varphi_{\omega}\right) \sin \left(\omega_{0} t\right)\right), \\
& k_{z}^{\prime}=\cos ^{2}\left(\theta_{\omega}\right)+\cos \left(\omega_{0} t\right) \sin ^{2}\left(\theta_{\omega}\right) .
\end{aligned}
$$

The helicity spinor transforms as follows,

$$
\begin{aligned}
a_{+}\left(k \hat{e}_{z}\right) & =\left(\begin{array}{l}
1 \\
0
\end{array}\right) \rightarrow\left(\cos \left(\frac{1}{2}|\vec{\varphi}|\right) \mathbf{1}_{2 \times 2}+\mathrm{i} \frac{\sin \left(\frac{1}{2}|\vec{\varphi}|\right)}{|\vec{\varphi}|} \vec{\sigma} \cdot \vec{\varphi}\right)\left(\begin{array}{l}
1 \\
0
\end{array}\right) \\
& =\left(\begin{array}{c}
\cos \left(\frac{1}{2} \omega_{0} t\right)+\mathrm{i} \cos \left(\theta_{\omega}\right) \sin \left(\frac{1}{2} \omega_{0} t\right) \\
\mathrm{i} \mathrm{e}^{\mathrm{i} \varphi_{\omega}} \sin \left(\theta_{\omega}\right) \sin \left(\frac{1}{2} \omega_{0} t\right)
\end{array}\right)=\mathrm{e}^{\mathrm{i} \arctan \left(\cos \left(\theta_{\omega}\right) \tan \left(\frac{1}{2} \omega_{0} t\right)\right)} a_{+}\left(\vec{k}^{\prime}\right) .
\end{aligned}
$$

The phase factor simplifies to $\exp \left(\mathrm{i} \omega_{0} t / 2\right)$ for $\theta_{\omega}=0$. From Eq. (A8), one might otherwise conclude that the lower

component (negative helicity) of the spinor $a_{+}(\vec{k})$ gets populated, upon a Lorentz transformation involving a rotation, with the amplitude

$$
\left|\mathrm{i} \mathrm{e}^{\mathrm{i} \varphi_{\omega}} \sin \left(\theta_{\omega}\right) \sin \left(\frac{1}{2} \omega_{0} t\right)\right|^{2}=\sin ^{2}\left(\theta_{\omega}\right) \sin ^{2}\left(\frac{1}{2} \omega_{0} t\right)
$$

and interpret this as a rotation-induced helicity flip [see Eq. (7) of Ref. 56]]. However, this is not the case, because one needs to interpret the transformed helicity spinor in terms of the transformed wave function [factor exp(i $\left.\vec{k}^{\prime} \cdot \vec{r}^{\prime}\right)$ ], where it reproduces the positive-helicity spinor $a_{+}\left(\vec{k}^{\prime}\right)$, up to the phase factor exp[i $\left.\arctan \left(\cos \left(\theta_{\omega}\right) \tan \left(\frac{1}{2} \omega_{0} t\right)\right)\right]$. Finally, the bispinor transforms as follows,

$$
\begin{gathered}
u_{+}\left(k \hat{e}_{z}\right) \mathrm{e}^{\mathrm{i} k z-\mathrm{i} E t}=\left(\begin{array}{c}
\sqrt{\frac{E+m}{2 m}}\left(\begin{array}{l}
1 \\
0
\end{array}\right) \\
\sqrt{\frac{E-m}{2 m}}\left(\begin{array}{l}
1 \\
0
\end{array}\right)
\end{array}\right) \rightarrow S(\Lambda) u_{+}\left(k \hat{e}_{z}\right) \mathrm{e}^{\mathrm{i} \vec{k}^{\prime} \cdot \vec{r}^{\prime}-\mathrm{i} E t} \\
=\mathrm{e}^{\mathrm{i} \arctan \left(\cos \left(\theta_{\omega}\right) \tan \left(\frac{1}{2} \omega_{0} t\right)\right)}\left(\begin{array}{c}
\sqrt{\frac{E+m}{2 m}} a_{+}\left(\vec{k}^{\prime}\right) \\
\sqrt{\frac{E-m}{2 m}} a_{+}\left(\vec{k}^{\prime}\right)
\end{array}\right) \mathrm{e}^{\mathrm{i} \vec{k}^{\prime} \cdot \vec{r}^{\prime}-\mathrm{i} E t} .
\end{gathered}
$$

(The energy is invariant under the rotation.) This formula confirms once more that a rotation of the reference system cannot induce a helicity flip of the neutrino; our arguments are in full agreement with the discussion following Eq. (2.341) in Sec. 2.10.1 of Ref. [2] but is at variance with the conclusions recently reached in Ref. [56]. One cannot avoid a Lorentz boost (as opposed to a rotation), as described in Sec. III to induce the elusive helicity reversal of the Dirac bispinor.

\section{Appendix B: Neutrinos and Generalized Dirac Hamiltonians}

Having clarified our alternative suppression mechanism for right-handed neutrinos, described by the tachyonic Dirac equation. we should now also include a few remarks on neutrino mass mixing in the tachyonic sector. The possibility of three-generation neutrino flavour-mass mixing has been indicated in Refs. [57, 58, and parameterized in the PMNS matrix. Reviews on the theory of massive neutrinos can be found in Refs. [5, 59, 60]. A discussion of the mixing matrix in concise form has been given in Ref. [61]. One usually assumes that the neutrino flavour eigenstate $\nu_{\ell L}(x)$ with $\ell=e, \mu, \tau$ is a linear superposition of the mass eigenstates $\nu_{i L}$ with $i=1,2,3$ as follows,

$$
\nu_{\ell L}(x)=\sum_{i=1}^{3} U_{\ell i} \nu_{i L}(x) .
$$


If CPT symmetry holds and if there are no highly non-standard interactions affecting neutrinos and anti-neutrinos differently, then the antineutrino eigenstates are mixed with the complex conjugate matrix,

$$
\bar{\nu}_{\ell L}(x)=\sum_{i=1}^{3} U_{\ell i}^{*} \bar{\nu}_{i L}(x)
$$

The tachyonic Dirac equation is obtained from the ordinary Dirac equation based on the replacement $m \rightarrow \gamma^{5} m$. One could thus introduce flavour-mass mixing matrix in the tachyonic sector. In particular, the result [60]

$$
P\left(\nu_{\ell} \rightarrow \nu_{\ell^{\prime}}\right)=\delta_{\ell \ell^{\prime}}-2 \operatorname{Re} \sum_{i>k} U_{\ell^{\prime} i} U_{\ell i}^{*} U_{\ell^{\prime} k}^{*} U_{\ell k}\left[1-\exp \left(-\mathrm{i} \frac{\Delta m_{k i}^{2} L}{2 E}\right)\right]
$$

for neutrino oscillations in a baseline experiment with length $L$, remains valid in the tachyonic sector, provided one assumes a single, tachyonic mass term of the form $\gamma^{5} m$ and parameterizes the mixing matrix accordingly.

With an infinitesimally superluminal neutrino, it is very hard to send information into the past, because of the smallness of neutrino interaction cross sections at low energy. Colloquially speaking, the dilemma is that high-energy tachyonic neutrinos approach the light cone and travel only infinitesimally faster than light itself $\left(E=\sqrt{\vec{p}^{2} c^{2}+m^{2} c^{4}} \approx\right.$ $|\vec{p}| c$ for high energy). Their interaction cross sections may be sufficiently large to allow for good detection efficiency but this is achieved at the cost of sacrificing the speed advantage. Low-energy tachyonic neutrinos may a substantially faster than light but their interaction cross sections are small and the information sent via them may be lost. A more quantitative argument is given in Ref. 62]. If we raise the impossibility to send information into the past to a postulate, then tachyonic spin-1/2 particles, if they exist, have to be very light.

According to the Feynman prescription, the propagator of the quantized Dirac field has an advanced, strictly speaking acausal part which describes antiparticles moving backward in time, and it also has a causal, retarded part, describing particles moving forward in time. However, the only physically relevant amplitudes predicted by theory are elements of the scattering matrix ( $S$-matrix). Antiparticles moving backward in time are reinterpreted as entities moving forward in time, with all kinetic variables (energy, momentum) changing sign [63, 64]. This is a manifestation of the principle of reinterpretation. The transition amplitude predicted by $S$ matrix theory connects two space-time points, and the time coordinate of one of the events happens to be earlier than the other. Particles, seen by an observer, always move forward in time because it is always possible to identify the "earlier" event whose time coordinate is less than that of the other event. If the particles described by the theory are subluminal (move slower than light), then this reinterpretation is undisputed within the community and forms one the core foundations of experimentally verified quantum field theory. This concept has been generalized to superluminal particles 52].

The compatibility of faster-than-light travel with the axioms of special relativity has been discussed in Refs. 51 55, 65, 66]. Via a geometric construction (Minkowski diagram), one can show that a superluminal velocity remains superluminal if one changes Lorentz frames. In particular, the Einstein velocity addition theorem remains valid in the superluminal world [51, 52]. Nimtz and coworkers [67 69] claim to have demonstrated in their (disputed) experiments that electromagnetic signal propagation with up to four times of the speed of light is possible if one is willing to accept exponential damping of the signal (tunneling effect), i.e., over short distances.

The quantum field theory of superluminal particles is plagued with a series of problems, and it was soon realized that not all of the so-called Osterwalder-Schrader axioms 70] can be retained if one tries to incorporate tachyonic particles into field theory. In a series of recent papers [11-13], we have used the concept of a Lorentz non-invariant vacuum state, breaking one of the Osterwalder-Schrader [70] axioms, but we have retained absolute conformity with Lorentz covariance and the special theory of relativity. Also, we have offered an alternative picture ("re-reinterpretation") in Sec. 4 of Ref. [11]) where we work with a Lorentz-invariant vacuum while transforming only the space-time argument of the creation and annihilation operators, but not, as it would seem necessary otherwise, some of the annihilation operators of the tachyonic field into creation operators and vice versa. The Hamiltonians describing faster-than-light particles are pseudo-Hermitian. The concept of pseudo-Hermiticity was introduced in Ref. [34]. Coincidentally, the only particle which is a candidate for superluminality, the neutrino, has been observed in only one helicity (lefthanded), while antineutrinos come in right-handed helicity. This is precisely what our anticommutator relation (37) predicts, and postulating this anticommutator is the only possibility we found in order to complete the spinor sum in Eq. (35), where the prefactor $(-\sigma)$ is necessary in order to obtain the positive-energy and negative-energy projectors. No further alterations are necessary for the theory of weak interactions because Lorentz invariance is fully conserved. In principle, the numerical value of the tachyonic mass term could be determined using more precise experiments on neutrino flight times or by looking at the end point of the tritium beta decay experiments more accurately.

If we assume that if the neutrino is superluminal, then the neutrino is not equal to its own antiparticle, and neutrinoless double beta decay is forbidden. While this conclusion somewhat depends on the precise equation proposed for the description of the neutrino, none of the currently proposed equations [7, 11 13, 71, 72] is charge conjugation 
invariant, so that there are no charge-conjugation-invariant Majorana solutions of the tachyonic spin- $1 / 2$ equations. We recall that experimental evidence for the observation of neutrinoless double beta decay [73 79] is disputed, and recent measurements set even stricter bounds for the magnitude of the Majorana mass terms (for an overview see pp. 176 ff. of Ref. [4]). Relatively recent results include data from Cuoricino (see Ref. [19]), from the EXO-200 collaboration [20], from KamLAND-Zen [21] and GERDA [22].

\section{Appendix C: Majorana Equation}

Let $\omega(x)$ denote a two-component spinor amplitude. In the conventions of Ref. 6], where the chiral representation of the Dirac algebra is employed, the Majorana equation reads [see Eq. (6.25) of Ref. [6]]

$$
\bar{\sigma}^{\mu} \partial_{\mu} \omega(x)+m \sigma^{2} \omega^{*}(x)=0 .
$$

Here, $\bar{\sigma}^{\mu}=(1,-\vec{\sigma})$, and the $y$ component of the $\vec{\sigma}$ vector is denoted as $\sigma^{2}$. This is a two-component equation which clearly cannot be solved by a plane-wave ansatz because the first term would involve a factor $\exp (-\mathrm{i} k \cdot x)$, whereas the second one, which involves the complex conjugate $\omega^{*}(x)$, would go as $\exp (\mathrm{i} k \cdot x)$. Consequently, in the field operator of the Majorana particle given in Eq. (18), the plane-wave spinor wave functions multiplying the creation and annihilation operators in the Fourier decomposition are not plane-wave eigenstates of the Majorana equation.

In Ref. [80], it is stated that the mass eigenstates of the Majorana neutrino are not plane waves but are of the form $\nu_{L}+\nu_{L}^{c}$, i.e., superpositions of left- and right-handed states [specifically, see Eq. (3) of Ref. [80]]. This is permissible if the neutrino is its own antiparticle. In this context, we also recall that the charge-conjugation invariant solutions of the massless Dirac equation,

$$
\Psi_{+}(x)=u_{+}(\vec{k}) \mathrm{e}^{-\mathrm{i} k \cdot x}+v_{-}(\vec{k}) \mathrm{e}^{\mathrm{i} k \cdot x}, \quad C \bar{\Psi}_{+}^{\mathrm{T}}(x)=\Psi_{+}(x)
$$

and

$$
\Psi_{-}(x)=u_{-}(\vec{k}) \mathrm{e}^{-\mathrm{i} k \cdot x}+v_{+}(\vec{k}) \mathrm{e}^{\mathrm{i} k \cdot x}, \quad C \bar{\Psi}_{-}^{\mathrm{T}}(x)=\Psi_{-}(x),
$$

are not plane waves with a definite four-momentum, but superpositions of plane waves which travel in opposite directions. Charge conjugation invariance holds because of the relation $C \bar{u}_{ \pm}(\vec{k})=v_{\mp}$ with $C=\mathrm{i} \gamma^{2} \gamma^{0}$ (in the standard representation). So, an outgoing Majorana neutrino in a scattering process, described by a wave with a definite fourmomentum, momentum cannot simultaneously be in an energy eigenstate of the Majorana equation. By contrast, all eigenspinors of the Dirac equation (with scalar or pseudo-scalar mass terms) simultaneously constitute energy and momentum eigenstates of the Dirac Hamiltonian, in full compatibility with unperturbed electrons, positrons, heavy leptons, photons, heavy gauge bosons, and quarks, which are all described by plane-wave states proportional to $\mathrm{e}^{-\mathrm{i} k \cdot x}$.

[1] B. Kayser, F. Perrier, and F. Gibrat-Debu, The Physics of Massive Neutrinos (World Scientific, Singapore, 1989).

[2] C. Giunti and C. W. Kim, Fundamentals of Neutrino Physics and Astrophysics (Oxford University Press, Oxford, UK, 2007).

[3] V. Barger, D. Marfatia, and K. Whisnant, The Physics of Neutrinos (Princeton University Press, Princeton, NJ, 2012).

[4] K. Zuber, Neutrino Physics (CRC Press, Boca Raton, FL, 2012).

[5] W. Rodejohann, Neutrinoless double beta decay and neutrino physics, J. Phys. G 39, 124008 (2012).

[6] P. B. Pal, Majorana and Weyl Fermions, Am. J. Phys. 79, 485-498 (2011).

[7] A. Chodos, A. I. Hauser, and V. A. Kostelecky, The Neutrino as a Tachyon, Phys. Lett. B 150, $431-435$ (1985).

[8] A. Chodos, V. A. Kostelecky, R. Potting, and E. Gates, Null experiments for neutrino masses, Mod. Phys. Lett. A 7, 467-476 (1992).

[9] V. A. Kostelecky, Mass bounds for spacelike neutrinos, Published in Topics on Quantum Gravity and Beyond: Essays in Honor of Louis Witten on his retirement, F. Mansouri and J. J. Scanio, eds. (World Scientific, Singapore, 1993).

[10] A. Chodos and V. A. Kostelecky, Nuclear null tests for space-like neutrinos, Phys. Lett. B 336, 295-302 (1994).

[11] U. D. Jentschura and B. J. Wundt, Localizability of Tachyonic Particles and Neutrinoless Double Beta Decay, Eur. Phys. J. C 72, 1894 (2012) [13 pages].

[12] U. D. Jentschura and B. J. Wundt, Pseudo-Hermitian Quantum Dynamics of Tachyonic Spin-1/2 Particles, J. Phys. A 45, 444017 (2012) [19 pages]. 
[13] U. D. Jentschura and B. J. Wundt, From Generalized Dirac Equations to a Candidate for Dark Energy, ISRN High Energy Physics 2013, 374612 (2013) [21 pages].

[14] V. A. Kostelecky and M. Mewes, Neutrinos with Lorentz-violating operators of arbitrary dimension, Phys. Rev. D 85, 096005 (2012).

[15] J. C. Pati and A. Salam, Lepton number as the fourth color, Phys. Rev. D 10, 275-289 (1974).

[16] G. Senjanovic and R. N. Mohapatra, Exact left-right symmetry and spontaneous violation of parity, Phys. Rev. D 12, 1502 (1975).

[17] R. N. Mohapatra and G. Senjanovic, Neutrino Mass and Spontaneous Parity Nonconservation, Phys. Rev. Lett. 44, 912-915 (1979).

[18] S. M. Bilenky and C. Giunti, Neutrinoless double-beta decay. A brief review, Mod. Phys. Lett. A 27, 1230015 (2012).

[19] L. Gironi, Cryogenic bolometer crystals for rare event searches, Eur. Phys. J. Plus 127, 84 (2012).

[20] M. Auger et al. [EXO-200 Collaboration], Search for Neutrinoless Double-Beta Decay in ${ }^{136}$ Xe with EXO-200, Phys. Rev. Lett. 109, 032505 (2012).

[21] A. Gando et al. [KamLAND-Zen Collaboration], Limit on Neutrinoless $\beta \beta$ Decay of ${ }^{136}$ Xe from the First Phase of KamLAND-Zen and Comparison with the Positive Claim in ${ }^{76}$ Ge, Phys. Rev. Lett. 110, 062502 (2013).

[22] C. Macolino et al. [GERDA Collaboration], Results on neutrinoless double beta decay from GERDA phase I, Mod. Phys. Lett. A 29, 143001 (2013).

[23] An illustrative presentation by R. Slansky, S. Raby, T. Goldman, G. Garvey and N. G. Cooper is available for download at the URL http://brock.physik.uni-bonn.de/ brock/teaching/vtp_ws0304/doc/oscillating_neutrino.pdf.

[24] A. S. Goldhaber and M. Goldhaber, The neutrinos elusive helicity reversal, Phys. Today 64, 40-43 (2011).

[25] M. P. Fewell, Question \# 76. Neutrino Mass and Helicity, Am. J. Phys. 66, 751 (1998).

[26] B. R. Holstein, Answer to Question \# 76. Neutrino Mass and Helicity, Am. J. Phys. 66, 1045 (1998).

[27] V. B. Berestetskii, E. M. Lifshitz, and L. P. Pitaevskii, Quantum Electrodynamics, 2 ed. (Pergamon Press, Oxford, UK, 1982).

[28] W. O. Straub has presented a related derivation and an illustrative overview at the URL http://www.weylmann.com/weyllorentz.pdf

[29] V. Mourik, K. Zuo, S. M. Frolov, S. R. Plissard, E. P. A. M. Bakkers, and L. P. Kouwenhoven, Signatures of Majorana Fermions in Hybrid Superconductor-Semiconductor Nanowire Devices, Science 336, 1003-1007 (2012).

[30] J. Casanova, C. Sabin, J. Leo, I. L. Egusquiza, R. Gerritsma, C. F. Roos, J. J. Garcia-Ripoll, and E. Solano, Quantum Simulation of the Majorana Equation and Unphysical Operations, Phys. Rev. X 1, 021018 (2011).

[31] B. Kayser and R. E. Shrock, Distinguishing between Dirac and Majorana Neutrinos in Neutral-Current Reactions, Phys. Lett. B 112, 137-142 (1981).

[32] L. F. Li and F. Wilczek, Physical processes involving Majorana neutrinos, Phys. Rev. D 25, 143-148 (1982).

[33] B. Kayser, Majorana neutrinos and their electromagnetic properties, Phys. Rev. D 26, 1662-1670 (1982).

[34] W. Pauli, On Dirac's new method of field quantization, Rev. Mod. Phys. 15, 175-207 (1943).

[35] C. M. Bender and S. Boettcher, Real Spectra in Non-Hermitian Hamiltonians Having PT-Symmetry, Phys. Rev. Lett. 80, 5243-5246 (1998).

[36] C. M. Bender, S. Boettcher, and P. N. Meisinger, PT-Symmetric Quantum Mechanics, J. Math. Phys. 40, 2201-2229 (1999).

[37] C. M. Bender and E. J. Weniger, Numerical evidence that the perturbation expansion for a non-Hermitian $\mathcal{P} \mathcal{T}$-symmetric Hamiltonian is Stieltjes, J. Math. Phys. 42, 2167-2183 (2001).

[38] A. Mostafazadeh, Pseudo-Hermiticity versus $\mathcal{P} \mathcal{T}$-symmetry: The necessary condition for the reality of the spectrum of a non-Hermitian Hamiltonian, J. Math. Phys. 43, 205-214 (2002).

[39] A. Mostafazadeh, Pseudo-Hermiticity versus $\mathcal{P} \mathcal{T}$-Symmetry. II. A complete characterization of non-Hermitian Hamiltonians with a real spectrum, J. Math. Phys. 43, 2814-2816 (2002).

[40] A. Mostafazadeh, Pseudo-Hermiticity and Generalized PT- and CPT-Symmetries, J. Math. Phys. 44, 974-989 (2003).

[41] U. D. Jentschura, A. Surzhykov, and J. Zinn-Justin, Unified Treatment of Even and Odd Anharmonic Oscillators of Arbitrary Degree, Phys. Rev. Lett. 102, 011601 (2009).

[42] U. D. Jentschura, A. Surzhykov, and J. Zinn-Justin, Multi-Instantons and Exact Results III: Unified Description of the Resonances of Even and Odd Anharmonic Oscillators, Ann. Phys. (N.Y.) 325, 1135-1172 (2010).

[43] C. Gattringer and C. B. Lang, Quantum Chromodynamics on the Lattice: An Introductory Presentation (Lecture Notes in Physics Vol. 788) (Springer, Heidelberg, 2009).

[44] C. Itzykson and J. B. Zuber, Quantum Field Theory (McGraw-Hill, New York, 1980).

[45] J. H. Noble, M. Lubasch, and U. D. Jentschura, Generalized Householder Transformations for the Complex Symmetric Eigenvalue Problem, Eur. Phys. J. Plus 128, 93 (2013) [13 pages].

[46] H. F. Jones, Use of Equivalent Hermitian Hamiltonian for PT-Symmetric Sinusoidal Optical Lattices, J. Phys. A 44, $345302(2011)$.

[47] A. Szameit, M. C. Rechtsman, O. Bahat-Treidel, and M. Segev, PT-Symmetry in honeycomb photonic lattices, Phys. Rev. A 84, 021806(R) (2011).

[48] P. Ehrenfest, Gleichförmige Rotation starrer Körper und Relativitätstheorie, Phys. Z. 10, 918 (1909).

[49] L. Herrera, The relativistic transformation to rotating frames, Nuovo Cim. B 115, 307-318 (2000).

[50] M. Nouri-Zonoz, H. Ramezani-Aval, and R. Gharechahi, On rotation and rotating frames: Franklin transformation and its modification, e-print arXiv:1208.1913 [gr-qc].

[51] O. M. P. Bilaniuk, V. K. Deshpande, and E. C. G. Sudarshan, "Meta” Relativity, Am. J. Phys. 30, 718-723 (1962). 
[52] O.-M. Bilaniuk and E. C. G. Sudarshan, Causality and Space-like Signals, Nature (London) 223, 386-387 (1969).

[53] E. Recami, Superluminal waves and objects: an overview of the relevant experiments, J. Phys. Conf. Ser. 196, 012020 (2009).

[54] O. M. Bilaniuk, Tachyons, J. Phys. Conf. Ser. 196, 012021 (2009).

[55] S. K. Bose, Aspects of Tachyon theory, J. Phys. Conf. Ser. 196, 012022 (2009).

[56] D. V. Ahluwaila and C.-Y. Lee, Gamma-ray bursts and the relevance of rotation-induced neutrino sterilization, Phys. Lett. B 719, 218-219 (2013).

[57] B. Pontecorvo, Mesonium and anti-mesonium, Zh. Éksp. Teor. Fiz. 33, 549-551 (1957), [Sov. Phys. JETP 6, 429 (1958)].

[58] Z. Maki, M. Nakagawa, and S. Sakata, Remarks on the Unified Model of Elementary Particles, Prog. Theor. Phys. (Kyoto) 28, 870 (1962).

[59] S. M. Bilenky and S. T. Petcov, Massive neutrinos and neutrino oscillations, Rev. Mod. Phys. 59, 671-754 (1987), [Errata Rev. Mod. Phys. 60, 575 (1988), and Rev. Mod. Phys. 61, 169 (1989)].

[60] S. M. Bilenky, C. Giunti, and W. Grimus, Phenomenology of neutrino oscillations, Prog. Part. Nucl. Phys. 43, 1-86 (1999).

[61] S. M. Bilenky, Majorana Neutrino Mixing, J. Phys. G 32, R127-R149 (2006).

[62] U. D. Jentschura, D. Horváth, S. Nagy, I. Nándori, Z. Trócsányi, and B. Ujvári, Weighing the Neutrino, Int. J. Mod. Phys. E 23, 1450004 (2014).

[63] R. P. Feynman, The theory of positrons, Phys. Rev. 76, 749-759 (1949).

[64] E. Stückelberg, La mécanique du point materiel en théorie de relativité et en théorie des quanta, Helv. Phys. Acta 15, 23-37 (1942).

[65] G. Székely, The existence of superluminal particles is consistent with the kinematics of Einstein's special theory of relativity, e-print arXiv:1202.5790v1 [gen-ph], 2012.

[66] J. X. Madarász, M. Stannett, and G. Székely, Why Do the Relativistic Masses and Momenta of Faster-than-Light Particles Decrease as their Speeds Increase?, Symmetry, Integr. Geom.: Methods Appl. 10, 005 (2014).

[67] A. Enders and G. Nimtz, On superluminal barrier traversal, J. Physique I (Paris) 2, 1693-1698 (1992).

[68] G. Nimtz and A. A. Stahlhofen, Universal tunneling time for all fields, Ann. Phys. (Berlin) 17, 374-379 (2008).

[69] G. Nimtz, On virtual Phonons, Photons and Electrons, Found. Phys. 39, 1346-1355 (2009).

[70] K. Osterwalder and R. Schrader, Axioms for Euclidean Green's Functions, Commun. Math. Phys. 31, 83-112 (1973).

[71] T. Chang, A new Dirac-type equation for tachyonic neutrinos, e-print arXiv:hep-th/0011087

[72] T. Chang, Parity Violation and Neutrino Mass, e-print arXiv:hep-ph/0208239

[73] E. Fireman, A Measurement of the Half-Life of Double Beta-Decay from ${ }_{50} \mathrm{Sn}^{124}$, Phys. Rev. 75, 323-324 (1949).

[74] E. Fireman and D. Schwarzer, A Re-Investigation of the Double Beta-Decay from Sn ${ }^{124}$, Phys. Rev. 86, 451-453 (1952).

[75] H. V. Klapdor-Kleingrothaus, I. V. Krivosheina, A. Dietz, and O. Chkvorets, Search for neutrinoless double beta decay with enriched ${ }^{76}$ Ge in Gran Sasso 1990-2003, Phys. Lett. B 586, 198-212 (2004).

[76] H. V. Klapdor-Kleingrothaus and I. V. Krivosheina, The evidence for the observation of $0 \nu \beta \beta$ decay: The identification of $0 \nu \beta \beta$ events from the full spectra, Mod. Phys. Lett. A 21, 1547-1566 (2006).

[77] H. V. Klapdor-Kleingrothaus, A. Dietz, H. L. Harney, and I. V. Krivosheina, Evidence for neutrinoless double beta decay, Mod. Phys. Lett. A 16, 2409-2420 (2001).

[78] F. Feruglio, A. Strumia, and F. Vissani, Neutrino oscillations and signals in $\beta$ and $0 \nu 2 \beta$ experiments, Nucl. Phys. B 637, 345-377 (2002).

[79] Y. G. Zdesenko, F. A. Danevich, and V. I. Tretyak, Has neutrinoless double $\beta$ decay of ${ }^{76}$ Ge been really observed?, Phys. Lett. B 546, 206-215 (2002).

[80] B. Kayser, Are Neutrinos Their Own Antiparticles?, J. Phys. Conf. Ser. 173, 012013 (2009). 